\title{
ESTABLECIMIENTO DE LA RELACIÓN TERAPÉUTICA CON NIÑOS Y ADOLESCENTES CON DIFICULTADES DE REGULACIÓN Y VIOLENCIA ${ }^{1}$
}

\author{
Carmen Domingo Peña ${ }^{2}$ y Raúl Gutiérrez Sebastián ${ }^{3}$ \\ Instituto de Psicoterapia Relacional, Madrid / Zaragoza
}

\begin{abstract}
El presente texto pretende desarrollar ciertas reflexiones en torno a la necesidad de pensar el momento de establecimiento de la relación con niños y adolescentes violentos desde el inicio, incluso, de construcción de la demanda con los mismos, clarificando así un espacio de relación congruente y explicito, desde una actitud curiosa y compartida por el co-construir un espacio de mutualidad relacional nutricio para todos. Así mismo, consideramos la importancia de contemplar a los agentes adultos referentes en sus vidas con el propósito de generalizar cambio y dar continuidad a un proceso de cambio emergido de una relación y posibilitando una maduración saludable extendiendo el proceso de cambio a otros contextos significativos para el menor y transcendiendo a la relación misma de ayuda inicial, favoreciendo así procesos de desvinculación desde la propia vinculación.
\end{abstract}

Palabras clave: Demanda, mentalización, mutualidad relacional, generalización del cambio

The purpose of this paper is encouraging some reflections over the first steps during the establishment of the therapeutic relationship with children and teenagers with a violence behavior and self-regulation problems. We will discuss the importance of building a clear demand with the patient that provides a congruent therapeutic space that allows a curious and shared experience cobuilt by a mutual relational experience between the therapist and the young patient. In addition, we need to have in mind their significant caregivers as an important part of the process so that the change could be generalize. The final goal is that children and teenager could live a new relational experience that makes a difference in the way of bonding giving way to new forms of relationship in other areas of their life allowing a more mature and adaptive development.

Key Words: Demand, mentalization, relational mutuality, generalization of change English Title: ESTABLISHING A THERAPEUTIC RELATIONSHIP WITH CHILDREN AND ADOLESCENTS WITH SELF-REGULATION AND VIOLENCE DIFFICULTIES

\section{Cita bibliográfica / Reference citation:}

Domingo Peña, C. y Gutiérrez Sebastián, R. (2020). Establecimiento de la relación terapéutica con niños y adolescentes con dificultades de regulación y violencia. Clínica e Investigación Relacional, 14 (2): 431-439. [ISSN 1988-2939] [Recuperado de www.ceir.info ] DOI: 10.21110/19882939.2020.140210

\footnotetext{
${ }^{1}$ Trabajo presentado en la VIIIa Reunión Bienal de IARPP-España, Sevilla, 18 y 19 Octubre de 2019

2 Psicóloga infanto-juvenil y de adultos en práctica privada. Miembro de IPR e IARPP. E-mail: carmendomingo@cop.es

3 Psicólogo de FAIM. Psicoterapeuta FEAP. Vicepresidente de ADP-CETS. E-mail: raulgs@cop.es
} 


\section{LA IMPORTANCIA DE LA CONSTRUCCIÓN DE LA DEMANDA}

Todo trabajo psicoterapéutico implica contemplar una serie de variables, personales y relacionales, que rodean al paciente que consulta. Sin embargo, cuando afrontamos el reto de trabajar con niños y adolescentes tenemos que contar con una variable inexistente en el trabajo con adultos, a saber, los propios progenitores. Es frecuente en estos casos encontrar una discrepancia entre la demanda de los padres y la de los propios niños o adolescentes.

Cuando Abeijón (2013) habla sobre la "patología de la demanda", plantea diversas formas de expresar un sufrimiento de manera encubierta, unas veces proyectada en el otro, otras de manera confusa, incongruente, incompleta y/o implícita, desde la cual, corremos el riesgo de construir un espacio de relación de ayuda sobre una base relacional "insegura" y confusa. La construcción de la demanda es la puerta de entrada a la relación de ayuda, y si no se concreta de manera congruente, explícita y clarificadora es probable que nos veamos sumergidos en la desorientación, e incluso en la inseguridad.

Entendemos la demanda como el punto en torno al cual se va a vertebrar la relación de ayuda, dado que no trabajamos "en el individudo", sino en ese "espacio de relación" entre consultante y terapeuta, y dado que precisamente es en la relación donde emergen las dificultades, acentuar un primer tiempo de clarificación y explicitación de la demanda puede ayudar a focalizar tanto el para qué de la relación, como posibles focos u objetivos a abordar inicialmente desde donde construir relación.

Abejión (2013) comenta que la propia demanda, va a contener características del demandante, así como del significado del problema que se plantea, la función del síntoma tanto en el sujeto como en el sistema familiar y el impacto que el mismo ocasiona tanto en el sujeto como en su entorno, emergiendo resonancias que consideramos, hay que atender, por lo que abordar la demanda y dedicar un tiempo a trabajar con las propias características de la misma, aparte de ofrecernos información relevante, ya es intervenir y construir el espacio de relación de ayuda.

En el caso que nos ocupa, el trabajo con niños/as y adolescentes con dificultades de regulación emocional y de relación, así como de violencia, la demanda suele construirse desde el eje parental, soliendo mostrar una demanda de manera "implícita" ("Ya imaginas por qué venimos, como habrás visto ya muchos casos, seguro que sabes cómo nos sentimos"), a veces "obligada" ("venimos porque nos lo indica fiscalía, servicios sociales... no es que necesitemos nada, pero si no venimos, nos llamarán la atención"), "dramática" ("No imaginas el sufrimiento que vivimos, ya no podemos vivir más así, necesitamos una solución inmediata, nos va a destrozar la vida") y "desplazada" ("nosotros estamos bien, si venimos aquí es por 
nuestro hijo, es quien realmente está mal y por eso hace lo que hace, a ver si aquí podéis ayudarle a que cambie").

Sin embargo, es necesario un trabajo "pre-tratamiento" que explicite el por qué y el para qué de la relación entre el niño/a o adolescente y el/la profesional, favoreciendo la emergencia de su propia demanda desde donde poder construir un espacio de relación que nutra y dote de seguridad para la creación de un vínculo disponible, predecible y permanente.

En estos primeros encuentros es necesario definir de forma clara los espacios, el encuadre, interno y externo (Estalayo, Rodríguez y Gutiérrez, 2016) y la vertebración de ambas demandas. Sin este trabajo previo de "construcción de la demanda", podemos llegar a sentirnos influenciados por el deseo de cambio y la angustia parental, interfiriendo con la sintonización con las necesidades de nuestro paciente.

Además, desde la propia clarificación y explicitación de una demanda propia desde donde construir relación, facilita también la posibilidad de establecer focos de intervención que den respuesta a necesidades propias del niño/adolescente. Ello favorecerá el protagonismo del niño/adolescente y su sensación de control sobre el espacio (Marlberg y cols., 2019), necesario para establecer una base segura de la relación.

\section{EL ESTABLECIMIENTO DE LA RELACIÓN}

De alguna manera comenzar un proceso terapéutico es algo semejante al momento en el que madre y bebé recién nacido se miran por primera vez. Ambos, han estado 9 meses fusionados, pero desconocen cómo será su relación tras el nacimiento; de la misma manera que se activa el imaginario tanto del terapeuta como del futuro joven paciente desde el momento de la demanda de atención hasta el primer encuentro.

Durante los primeros meses ambos aprenden a relacionarse, a descubrir sus ritmos y formas de expresarse. La madre responde sensiblemente a sus demandas y calibran recíprocamente sus interacciones (Sluzki, 2009) donde se intercambian pequeños gestos preverbales que resultarán cruciales para construir un vínculo seguro en la diada madre/padre-bebé. (Bebee y Lachmann, 2014). Algo similar a lo que sucede cuando comenzamos una terapia, vemos al infante o adolescente pero aún no nos conocemos. Los primeros meses se destinan a establecer esa primera relación, donde paciente y terapeuta comienzan a conocerse y a coconstruir juntos algo que no pertenece ni a uno ni a otro, sino que es de los dos, ese espacio compartido que conocemos como intersubjetividad.

Aprendemos a ver cómo se mueve cuando viene contento, cómo su gesto o actitud cambia cuando se enfada o frustra y nosotros aprendemos a responder con aquello que necesita de 
nosotros favoreciendo la puesta en palabras de elementos de actuación implícitos y procedimentales que permitan la simbolización de estados mentales (Bebee y Lachmann, 2014.)

Karen Maroda (2010) aborda la importancia de mantenernos conectados con el paciente y nuestras emociones como parte fundamental del proceso del psicoanálisis relacional. A lo largo de este trabajo vamos a abordar cómo trabajar la mutualidad y la mentalización como la vía para la regulación de las emociones presente en muchos casos de violencia filioparental, desde un encuadre de relación de base segura que permita la puesta en palabras de conflictos y emociones que antes se expresaban a través del cuerpo.

Abordaremos la importancia de ejercer la terapia desde la relación empática e intersubjetiva, sobre la base de confianza epistémica (Fonagy, 2016). Esto requiere analizar previamente cuál es la forma del paciente de pensarse a sí mismo y al otro, así como indagar sobre las relaciones que establece y los diversos contextos donde se desarrollan dichas relaciones. Establecer una relación de ayuda de apego seguro con alguien inseguro o desorganizado, implica explorar su capacidad reflexiva, los modelos operativos internos de apego desde donde construye su matriz relacional (Mitchell, 1993), para poder situarnos en la relación y "calibrar recíprocamente" una intervención de confianza que favorezca el proceso de cambio.

La violencia reactiva, a diferencia de la instrumental (Caprara y Pastorelli, 1993), aparece como respuesta ante lo incomprensible. La angustia que experimentan estos chicos/as ya sea en términos afectivos o racionales, internos o externos ocasiona una falla en la capacidad de mentalizarse a sí mismo o a otro que surge ante la inseguridad y falta de disponibilidado ante el miedo en casos de apego desorganizados-, que históricamente han experimentado en la relación con las figuras de apego significativas. Por eso, un requerimiento en la relación es ayudar al niño/a o adolescente a transitar dichas experiencias de incomprensibilidad e inseguridad, permitiendo volcar en la relación la angustia vivida, y así co-construir un espacio dialógico circular y reflexivo, entre la vivencia del paciente y la del terapeuta, que movilice a través de la experiencia relacional una comunicación intra e interpersonal (Gutiérrez, 2018), favoreciendo la integración de aspectos disociados del self (Bromberg, 2017).

\section{VIÑETA CLINICA}

Laura es una niña de 8 años. Acude a consulta acompañada por sus padres por presentar problemas de comportamiento graves dentro del domicilio en los momentos de frustración y de enfado. Cuando algo no le sale amenaza con hacerse daño o bien amenaza con objetos 
cortantes a sus padres verbalizando que les hará daño. En otros momentos arremete contra el mobiliario en un estado de enfado y disociación tal que los padres verbalizan alarmados que la cara se le cambia por completo y es como si no conocieran a su hija. Siempre acompañado de violencia verbal. Este comportamiento se circunscribe sólo al entorno familiar. Adecuado rendimiento académico con tendencia a la perfección, sintiendo gran vergüenza y frustración si comete un error. Socialmente se mueve en relaciones duales, siendo difícil la triangulación. Es una niña con tendencia a la introversión y en esta primera sesión familiar se le observa con gran tensión corporal y no es capaz de articular palabra.

\section{La demanda parental}

La construcción de la demanda de los padres, aunque con una base de realidad ante la gravedad de los estallidos de Laura, parte desde una demanda dramática. Verbalizan que no pueden más, que Laura va a dar con ellos y que su vida se ha convertido en un sufrimiento constante.

Esto nos da una pista de cómo los padres se están posicionando ante la violencia y el temor que infunde su propia hija. La representación mental que tienen de su hija es de alguien incontenible.

En estas primeras sesiones recojo dicha demanda y valido su sufrimiento así como su sensación de dolor al sentir que se alejan cada vez más de su hija. Les ofrezco también un espacio a ellos donde contener sus preocupaciones y angustias bien a través del correo intersesiones y cada tres semanas una sesión a solas con ellos.

A la vez que hago esto les devuelvo la necesidad de un trabajo cauteloso con su hija donde el foco inicial no serán los estallidos sino la construcción de un vínculo que le permita sentirse segura y no juzgada en la relación conmigo para poder abordar aquellos aspectos que transmiten tanta fragilidad.

Este movimiento permite, por un lado, contener la inseguridad y angustia de los padres, sintiendo que ahora somos tres los que tenemos en mente a Laura. Pueden apoyarse en alguien que les ha transmitido seguridad y calma en los momentos en los que se sienten desbordados. Por otro lado, disminuir el sentimiento de culpa de los padres y validar su sufrimiento e incluso el sentimiento de odio sin sentirse juzgados como padres permitió también que ellos soltarán un lastre que para muchos padres en esta situación les hace sentirse a la defensiva constantemente. 
Laura

Laura transmitía en su mirada y en su forma de estar en la consulta la primera sesión familiar una gran tensión y vergüenza al sentirse "descubierta" por sus padres cuando contaban los problemas. Al observarlo, paré y pude validar esos sentimientos dejando claro que no le juzgaría y que necesitaba entender qué pasaba para ayudarles a todos, puesto que veía que esta situación les estaba resultando dolorosa a toda la familia.

Pude observar como mi cara, mi voz y mi forma de dirigirme a ella sintonizaba con su sufrimiento y como en ese momento de tanta vergüenza al sentirse expuesta yo le validaba y a la vez terminaba mi intervención sobre no juzgarle con un guiño de ojo y una voz tranquila. Hubo desde el primer momento una experiencia de regulación emocional a través de la relación entre ambas.

No quiso venir a nuestra primera cita a solas, así que le escribí una carta donde volvía a dejar clara mi intención de ayuda lejos de cualquier juicio de valor. Finalmente pudimos finalizar la evaluación casi sin mediar palabras. No hicieron falta, el baile de gestos, miradas cómplices y validaciones emocionales (Bebee y Lachman, 1988) permitió ir poniendo palabras a su "inconsciente prerreflexivo" (Orange, Atwood y Stolorow, 2012) construyendo una intersubjetividad que en el futuro dará pie a la mentalización de sus estados emocionales y que le permitirá adquirir la habilidad de autorregulación.

Al finalizar la evaluación le devolví a ella lo que creía que estaba pasando. Entendí que su suspicacia y sentimiento de evidencia era tan grande que necesitaría saber que había alguien que le comprendía y que le podría sostener. Como las palabras no eran posibles y ella transmitía su malestar a través del cuerpo auné ambas cosas. Hicimos el experimento donde cuando te sujetan un brazo contra el cuerpo y tú haces una fuerza para subirlo, una vez que dejan de ejercer dicha fuerza el brazo se sube solo. Utilicé esto como metáfora de cómo su nivel de tensión era tan elevado que a veces se desbordaba de forma casi inesperada o ante situaciones banales. No dijo nada, sólo escuchó. Supe que me entendió porque al llegar a casa le hizo a su madre el mismo experimento. Ya tenía algo que "llevarse" fuera de la consulta y de nuestra relación que le permitiera comenzar a comprender qué le pasaba a Laura.

\section{Vínculos tempranos}

En este caso se confirman muchas de las teorías citadas en este texto que están relacionadas con los casos de violencia filio-parental. Existe un estilo de apego inseguro ambivalente. Observamos una madre con una gran fragilidad. Su forma de estar en el mundo transmite 
inseguridad, casi una sensación como de estado de alerta constante. Existe desde el momento del embarazo un estado de ánimo depresivo que se ha mantenido hasta la actualidad. Ya desde el embarazo experimentó grandes miedos, inseguridades y un nivel de estrés elevado. Esto nos permite pensar además cómo durante los meses de embarazo el nivel de cortisol podría ser elevado, lo cual se ha visto que puede estar relacionado con un temperamento más difícil del neonato.

El padre, por otro lado, también se muestra frágil y ambos con dificultades para establecer unos límites y una estructura adecuada que contuviera una personalidad tan compleja como la de Laura. Además, el papel del padre ha experimentado mayores momentos de ausencias.

Por otro lado, durante el primer año de vida y hasta la actualidad podemos pensar en cómo el bienestar emocional de esta madre ha sido tan frágil que esas primeras experiencias de autorregulación a través de la sintonía emocional, el espejamiento y lo que Stern llama attunement se han dado de forma incoherente. Podemos imaginar viendo el desbordamiento actual de ambos padres que las respuestas ante momentos de desregulación de Laura han podido ser ansiosas y sin la presencia de los límites necesarios, ante lo cual no ha podido internalizar la experiencia de tener un otro que me contenga (van der Kolk y Fisler, 1994).

Por eso, cuando ella y yo jugábamos en las primeras sesiones y al ver que iba perdiendo intentaba asuntarme. Me gritaba, daba golpes encima de la mesa, estornudaba cuando estaba concentrada y siempre mi respuesta era "aunque tu intentas asustarme, soy capaz de aguantar tus sustos. No me asustas." Porque en el trabajo con Laura necesitamos construir una experiencia de vinculación segura que le permita explorar su mundo emocional interno y poder tener la experiencia de contención y espejamiento que le permita una posterior autorregulación emocional.

\section{CONCLUSIÓN}

A modo de conclusión, consideramos relevante en el tratamiento con niños y adolescentes con dificultades de regulación y violencia, construir una demanda propia que vincule y vehicule la demanda parental, así como ofrecer una relación de ayuda que signifique una experiencia emocional novedosa en el marco de la mutualidad relacional a través del abordaje intersubjetivo, sintonizando con la dificultad expresada y balanceando procesos reflexivos y de mentalización que a su vez favorecen una relación de base segura, confiable y disponible. expresa. Este material es para uso científico y profesional exclusivamente y puede contener información clínica sensible. Los editores no se responsabilizan de los contenidos de los autores. Dirigir las consultas sobre derechos y autorizaciones a ceir@psicoterapiarelacional.es 
Además, y transversalmente a lo anteriormente planteado, es de especial importancia la generalización y externalización de la experiencia de base segura al contexto relacional inmediato donde se desarrolla madurativamente el infante/adolescente.

Como parte de los objetivos de un tratamiento suficientemente bueno, consideramos que se debería favorecer que el niño o adolescente tuviera la oportunidad de establecer o vivenciar otra opción de relación con la familia o agentes significativos en su vida (Estalayo, Rodríguez y Gutiérrez, 2017), que den continuidad a la experiencia de cambio en la relación terapéutica. Sin dicha generalización, sin un planteamiento integrativo que cuente con la participación de los progenitores o miembros significativos de su contexto relacional, resultará complicado madurar e integrar la experiencia de cambio en un proceso de maduración personal e interpersonal a través del ciclo vital.

\section{REFERENCIAS}

Abeijón, J.A. (2013). Patología de la demanda. Mosaico. Revista de la Federación Española de Asociaciones de Terapia Familiar, (54), 62- 75.

Bebbe, B y Lachmann, F. (1988). The Contribution of mother-infant mutual influence to the origins of self-and object representation. Psychoanalytic Psychology, 1988, 5(4), 305-337.

Bebbe, B. y Lachmann, F. (2014). The Origins of Attachment: Infant Research and Adult Treatment. New York: Routledge.

Bromberg, P. (2017). La sombra del Tsunami y el desarrollo de la mente relacional. Madrid. Colección Pensamiento Relacional

Caprara, G. V., \& Pastorelli, C. (1993). Early emotional instability, prosocial behaviour, and aggression: some methodological aspects. European Journal of personality, 7 (1), 19-36

Estalayo, A., Rodríguez, O. y Gutiérrez, R. (2016). El encuadre en contextos de intervención convivencial de protección de menores y justicia juvenil. Intervención psicoterapéutica en instituciones. Cuadernos de Psiquiatría y Psicoterapia del Niño y del Adolescente, (62), 33-42

Estalayo, A., Rodríguez, O. y Gutiérrez, R. (2017). Aplicaciones de la mentalización con Familias en Contextos de Protección y Justicia Juvenil. Clínica e Investigación Relacional, 11 (1): 110-133

Fonagy, P., Luyten, P., Allison, E. (2016). Petrificación epistémica y la restauración de la confiabilidad epistémica: Una nueva conceptualización del Trastorno Límite de la Personalidad y su tratamiento psicosocial. Clínica e Investigación Relacional, 10 (3): 587-629

Gutiérrez, R. (2018). La circularidad dialógica restaurativa de la angustia en el espacio de mutualidad relacional. Clínica e Investigación Relacional, 12 (1): 141-162.

Midgley, N., Ensink, K., Lindqvist, K., Marlberg, N. y Muller, N. (2019). Tratamiento basado en la mentalización para niños. Un abordaje de tiempo limitado. Desclée de Brouwer. Madrid 
Maroda, K. (2010). Psychodynamic Techniques: Working with Emotions in the Therapeutic Relationship. New York: Guilford.

Mitchell, S. A. (1993). Conceptos Relacionales en Psicoanálisis: una integración. Madrid: Siglo XXI.

Orange, D., Atwood, G. y Stolorow, R. (2012). Trabajando Intersubjetivamente: Contextualismo en la práctica clínica. Madrid: Colección Pensamiento Relacional.

Sluzki, C. (2009). Bebés difíciles, progenitores difíciles hacia un modelo basado en la calibración recíproca. Redes: revista de psicoterapia relacional e intervenciones sociales. (22), 11-27.

Van der Kolk, B y Fisler, R. (1994). Childhood abuse and neglect and loss of self-regulation. Bulletin of the Menninger Clinic, 58 (2), 145-169.

Original recibido con fecha: 30/10/2019 Revisado: 23/11/2019 Aceptado: 30/09/2020 